\title{
Far-infrared phonon-polariton dispersion probed by terahertz time-domain spectroscopy
}

\author{
Seiji Kojima* \\ Institute of Materials Science, University of Tsukuba, Tsukuba, Ibaraki 305-8573, Japan \\ Naoki Tsumura ${ }^{\dagger}$ and Mitsuo Wada Takeda \\ Department of Physics, Faculty of Science, Shinshu University, Matsumoto, Nagano 390-8621, Japan \\ Seizi Nishizawa \\ Cooperative Research Center, Faculty of Science, Shinshu University, Nagano, Nagano 380-8553, Japan
}

(Received 18 June 2002; published 6 January 2003)

\begin{abstract}
We report observations of the intensity and phase transmission spectra related to phonon-polariton propagation using coherent far-infrared radiation for a high-quality ferroelectric bismuth titanate crystal plate. In order to determine the polariton-dispersion relation, the phase delay was determined minutely as a function of the $\mathrm{THz}$ radiation frequency in the region between 3 and $100 \mathrm{~cm}^{-1}$. The anisotropy of polariton dispersion relation was also successfully determined on the $c$ plate simply by switching the polarization direction of an incident beam from $E \| a$ to $E \| b$. The observed polariton dispersion relations are consistently reproduced by the calculation using Kurosawa's formula.
\end{abstract}

DOI: 10.1103/PhysRevB.67.035102

PACS number(s): 78.47.+p, 71.36.+c, 77.84.Dy

\section{INTRODUCTION}

Dielectric properties of terahertz $(\mathrm{THz})$ regions are of great importance due to the rich physical and chemical properties in this range. Very recently the generation of coherent $\mathrm{THz}$ radiation by a femtosecond pulse laser has enabled the very promising technique of $\mathrm{THz}$ time-domain spectroscopy (THz-TDS). ${ }^{1-4}$ This technique has a better signal-to-noise ratio at frequency below $100 \mathrm{~cm}^{-1}$ than conventional farinfrared Fourier transform spectroscopy. It is based on ultrashort $\mathrm{THz}$ electromagnetic (EM) pulses, which are generated and detected by small photoconductive antennas driven by femtosecond laser pulses. This time-gaged coherent nature makes it possible to determine not only the transmission intensity but also its phase delay accurately. Furthermore, unique applications for dispersion relations for various excitations have become possible. In fact, for a photonic crystal, by measuring the phase delay as a function of radiation frequency, Wada and co-workers recently succeeded in determining the dispersion curves of EM waves related to the photonic band structure. ${ }^{5,6}$ However, such a phase delay related to other excitations has not yet been reported, for example, for a bulk phonon-polariton. Phonon-polaritons are universally known as propagating EM waves coupled to polar optical phonon.

In ferroelectric materials, soft optic modes responsible for ferroelectricity appear in the far-infrared region below 100 $\mathrm{cm}^{-1}$. The measurement of complex dielectric constants at $\mathrm{THz}$ frequency is very useful. Since ferroelectric soft modes are infrared active and they propagate as polaritons, the polariton dispersion in the far-infrared region gives very important information for both fundamental and technical problems in ferroelectrics. The ferroelectric materials of current interest are mostly perovskite families with oxygen octahedra. Their ferroelectricity originates dominantly from the instability of polar soft modes in a ferroelectric transition. Therefore, THz dynamics is very important for the study or characterization of ferroelectric properties. As one of the great technological achievements in the 1990s, oxide ferroelectric thin films have attracted a great deal of attention for use in nonvolatile memories. ${ }^{7,8}$ However, there are still some problems to overcome in order to measure very highfrequency dielectric properties accurately for thin films or for very thin plates. Consequently, an experimental method for determining $\mathrm{THz}$ dielectric properties is desired for fundamental and practical research.

In this paper, we report on a measurement of the intensity and phase of complex transmission using THz-TDS for a ferroelectric bismuth titanate crystal, which is a key material in ferroelectric memories. We then show that this nonlinear dispersion relation, determined from the phase delay, is consistently interpreted by taking account of phonon-polariton propagation in the crystal. The observed dispersion relation is in a good agreement with the calculated one.

\section{EXPERIMENT}

The sample used was a thin $c$-plate of a flux-grown bismuth titanate $\mathrm{Bi}_{4} \mathrm{Ti}_{3} \mathrm{O}_{12}$ (BIT) single crystal with the area of $15 \times 15 \mathrm{~mm}^{2}$ and a thickness of $d=0.23 \mathrm{~mm}$. Since the cleavage perpendicular to the $c$ axis is very strong, like mica crystals, very flat cleaved surfaces were used for measurements. The $\mathrm{THz}$ time domain spectrometer used ${ }^{5}$ is shown in Fig. 1. To reduce the absorption by water vapor, all the optics and the sample were placed in a vacuum chamber. Femtosecond pump pulses with a wavelength of $780 \mathrm{~nm}$ and a power of $20 \mathrm{~mW}$ were generated by a mode-locked compact fiber laser with a repetition rate of $80 \mathrm{MHz}$. The pump pulses were focused by an object lens onto the biased gap of the photoconductive antenna made of low-temperature grown GaAs. The emitted $\mathrm{THz}$ radiation was collimated and focused by a pair of off-axis paraboloidal mirrors onto the sample. The spot size of the $\mathrm{THz}$ beam on the sample is about $2 \mathrm{~mm}$ in diameter. The signals transmitted from the 


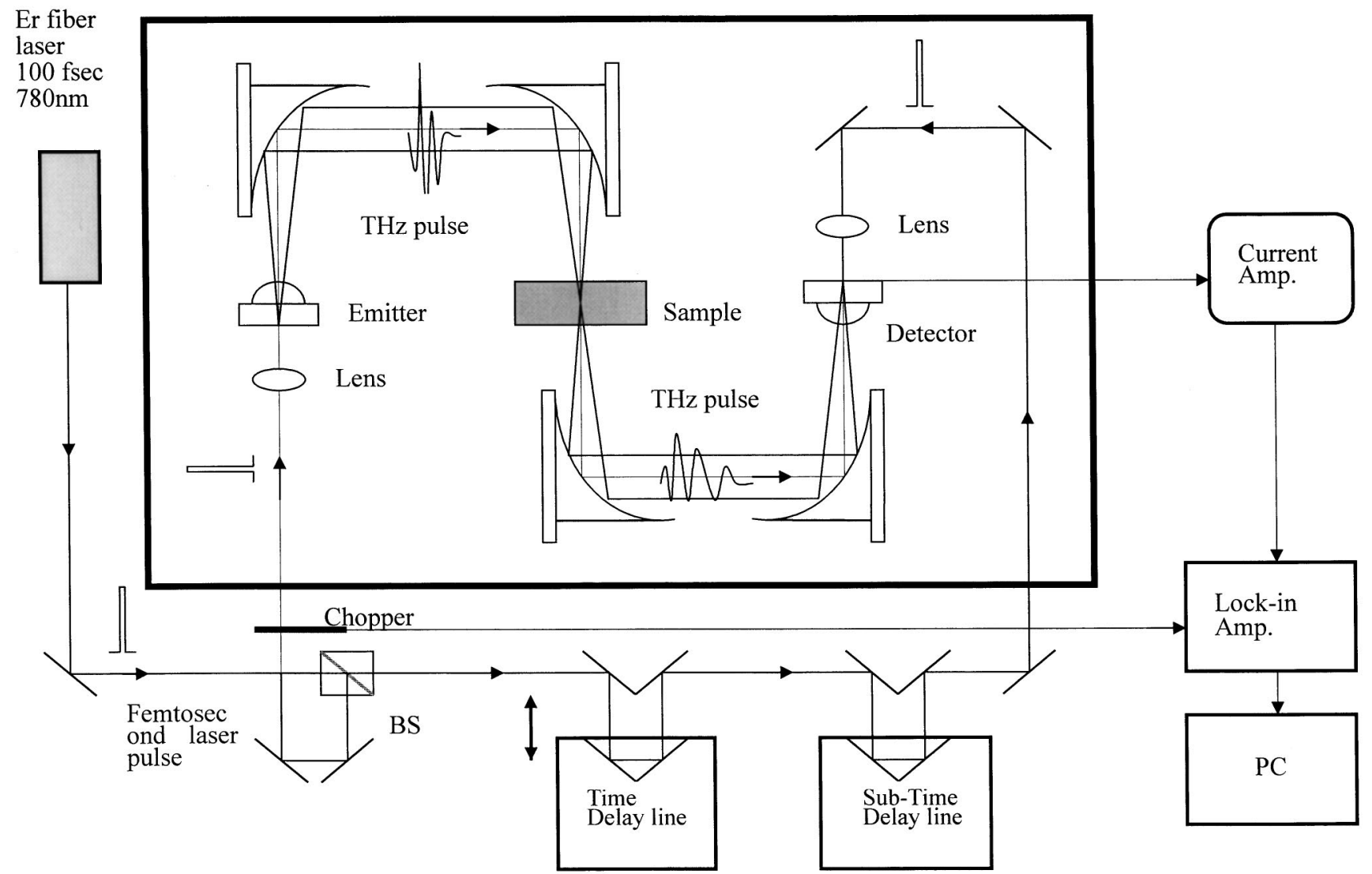

FIG. 1. Experimental setup of the THz time domain spectrometer.

sample were collimated again, and focused by another paraboloidal mirror onto a detector of a GaAs photoconductive antenna. The received signals were gated using incident pulses. An ammeter was used to measure the dc photocurrent induced by the transmitted radiation field from a sample. The waveform of transmitted EM pulses was determined by changing the delay time. The total acquisition time is $1 \mathrm{~h}$ and the number of scan is 30 .

\section{RESULTS}

BIT is one of the most important key materials in ferroelectric memories. BIT has a monoclinic-layered perovskite structure with the point group $m$ at room temperature. It undergoes a ferroelectric phase transition at $675^{\circ} \mathrm{C}$, and a hightemperature paraelectric phase is tetragonal with the point group $4 / \mathrm{mmm} .{ }^{9}$ The direction of spontaneous polarization is inclined at a small angle of about $4.5^{\circ}$ toward the monoclinic $a$ axis in the $a$ - $c$ plane. The $a$ - and $c$-axis components of the spontaneous polarization are 50 and $4 \mu \mathrm{C} / \mathrm{cm}^{2}$, respectively. In the ferroelectric phase, the displacive nature was observed with the underdamped soft optic mode at $28 \mathrm{~cm}^{-1}$ and at room temperature. ${ }^{10}$ Regarding optical phonon modes, $A^{\prime}(x, z)$ and $A^{\prime \prime}(y)$ modes are both infrared and Raman active where the mirror plane is perpendicular to the $y$ axis. The soft optic mode has an $A^{\prime}(x, z)$ symmetry where the coordinate $z$ is parallel to the $a$ axis.

Transmission spectra of the $c$ plate of BIT were observed at room temperature by using THz-TDS. $A^{\prime}(x, z)$ and $A^{\prime \prime}(y)$ modes are active when the light polarization is parallel to the $a$ axis $(E \| a)$ and $b$ axis $(E \| b)$, respectively. First, the timedomain $\mathrm{THz}$ signal for the polarization $E \| a$ was recorded as shown in Fig. 2. Second, this signal was converted into the frequency-domain power and phase, as shown in Fig. 3. Third, the transmission power was normalized by the reference one without the sample to be measured. The same procedure was carried out for the polarization $E \| b$. Figures 4(a) and 4(b) show the frequency dependence of transmission intensity and phase delay $(\phi / 2 \pi)$ in a range between 3 and 100 $\mathrm{cm}^{-1}$ for $E \| a$ and $E \| b$, respectively. It is found that $\phi$ vs $\omega$ shows nonlinear behavior near the gap edges at TO and LO

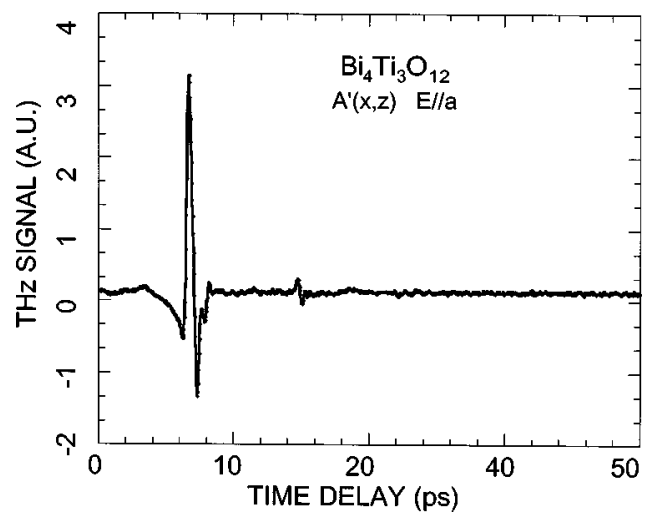

FIG. 2. The time-domain $\mathrm{THz}$ signals for the polarization $E \| a$. 


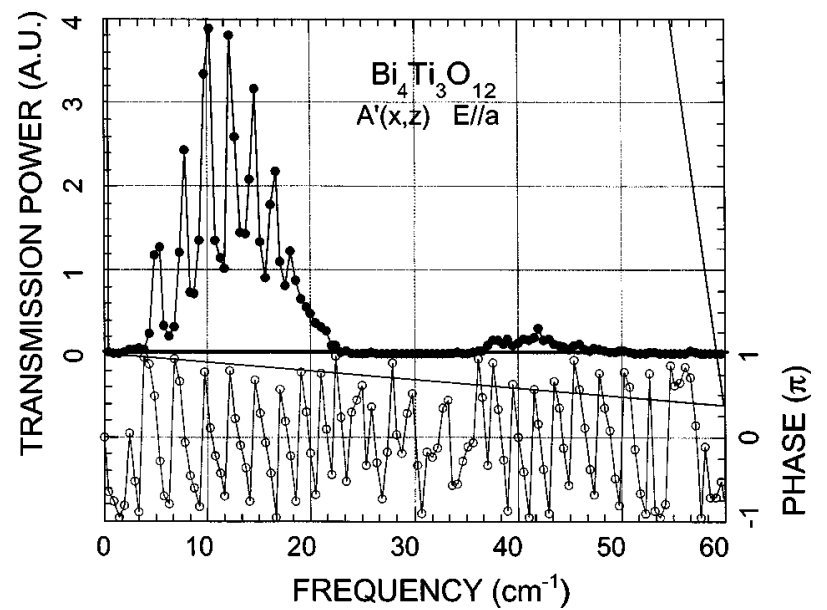

FIG. 3. The frequency-domain power and phase for the polarization $E \| a$.

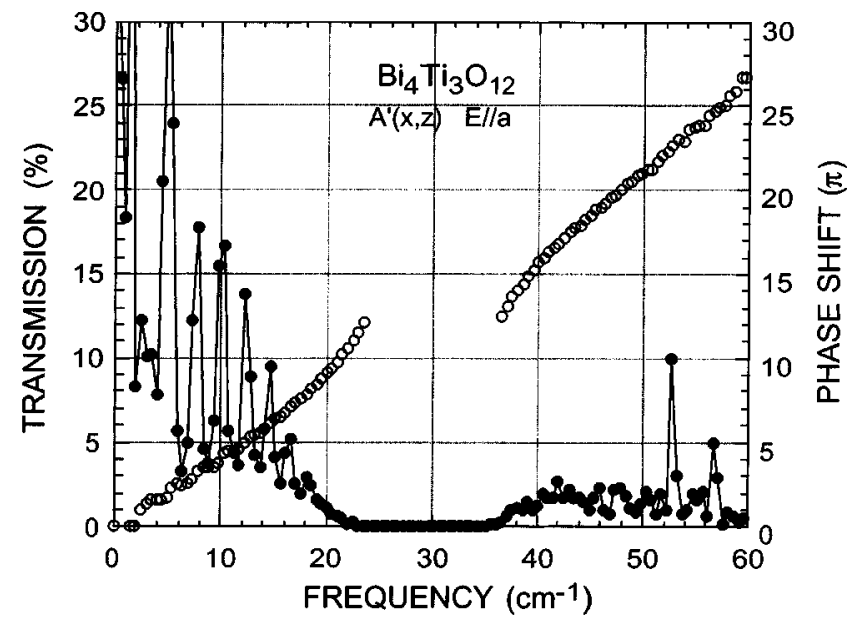

(a)

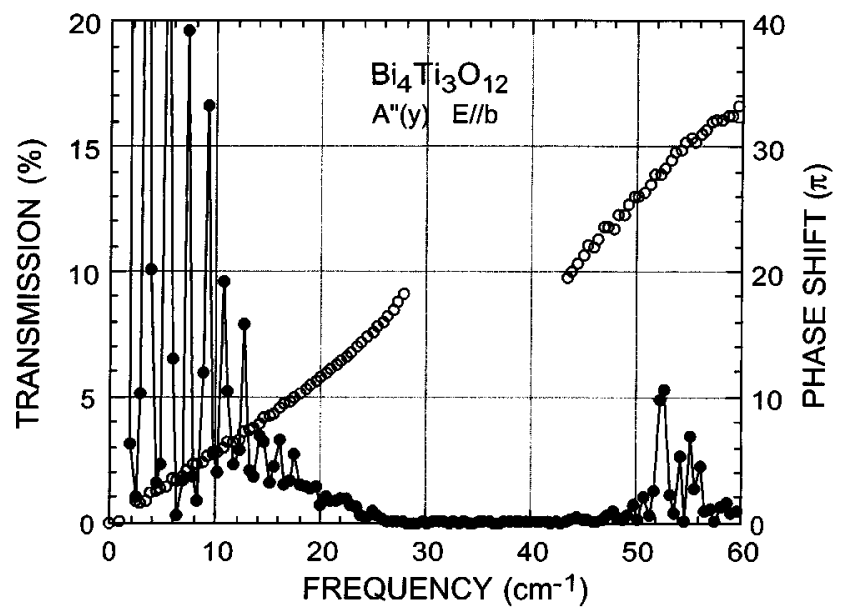

(b)

FIG. 4. Transmission intensity and phase shift spectra of the ferroelectric bismuth titanate crystal on the $c$ plate for the polarization along (a) the $a$ axis and (b) the $b$ axis. Closed and open circles denote the transmission intensity and phase shift, respectively.

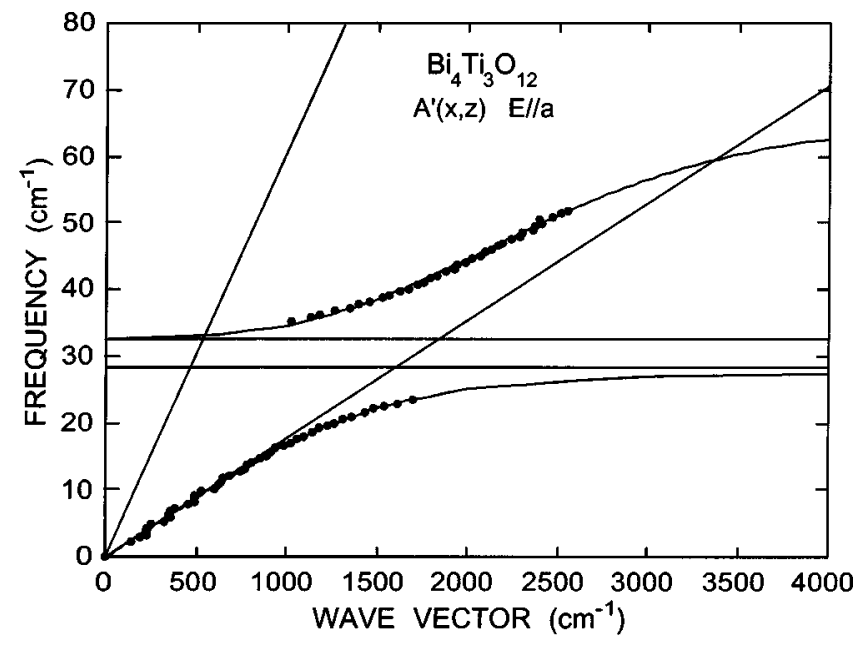

(a)

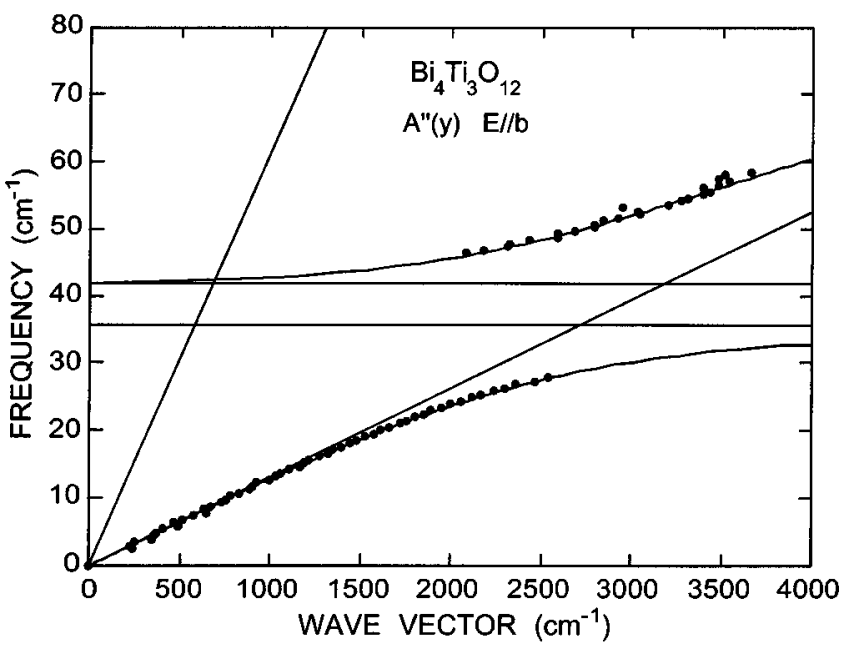

(b)

FIG. 5. Dispersion relations of phonon-polariton for a $c$-plate bismuth titanate crystal for the polarization along (a) the $a$ axis and (b) the $b$ axis. Closed circles denote observed values, and solid lines are calculated curves using Eq. (2).

mode frequencies. The increase of the gradient in the phase delay at the edges indicate the slowing of group velocity which is reasonable for the propagation of phonon-polariton. In order to determine the phonon-polariton dispersion relation, we calculated the wave vector $k(\omega)$ of the phononpolariton from the phase delay using the equation $\phi$ $=\{k(\omega)-\omega / c\} d$, where $c$ is the light velocity. Thus the dispersion relations were obtained as shown in Figs. 5(a) and $5(\mathrm{~b})$. For the polariton dispersion, $c^{2} k^{2} / \omega^{2}=\varepsilon(\omega){ }^{11,12}$ When the damping of phonon was negligible, a factorized form of $\varepsilon(\omega)$ was derived by Kurosawa. ${ }^{13}$ Consequently, the polariton-dispersion relation is given by

$$
\frac{c^{2} k^{2}}{\omega^{2}}=\varepsilon(\omega)=\varepsilon(\infty) \prod_{i=1}^{N} \frac{\omega_{\mathrm{LO} i}^{2}-\omega^{2}}{\omega_{\mathrm{TO} i}^{2}-\omega^{2}}
$$

Since the measured frequency is below $100 \mathrm{~cm}^{-1}$, we used the following approximation to fit the data: 
TABLE I. Fitting parameters of Eq. (2).

\begin{tabular}{lcc}
\hline \hline & $A^{\prime}(x, z): E \| a$ & $A^{\prime \prime}(y): E \| b$ \\
\hline$\omega_{\mathrm{TO} 1}$ & $28.0 \mathrm{~cm}^{-1}$ & $36.0 \mathrm{~cm}^{-1}$ \\
$\omega_{\mathrm{LO} 1}$ & $32.5 \mathrm{~cm}^{-1}$ & $42.0 \mathrm{~cm}^{-1}$ \\
$\omega_{\mathrm{TO} 2}$ & $68.0 \mathrm{~cm}^{-1}$ & $85.0 \mathrm{~cm}^{-1}$ \\
$\omega_{\mathrm{LO} 2}$ & $74.5 \mathrm{~cm}^{-1}$ & $98.5 \mathrm{~cm}^{-1}$ \\
$\varepsilon(\infty)$ & 6.76 & 6.76 \\
$\varepsilon(1)$ & 49.0 & 81.0 \\
$\varepsilon(0)$ & 79.2 & 149.0 \\
\hline \hline
\end{tabular}

$$
k(\omega)=\frac{\sqrt{\varepsilon(1)}}{c} \omega \prod_{i=1}^{2}\left\{\frac{\omega_{\mathrm{LO} i}^{2}-\omega^{2}}{\omega_{\mathrm{TO} i}^{2}-\omega^{2}}\right\}^{1 / 2},
$$

where we renormalize the contribution of the modes $i \geqslant 3$ to the fitting parameter $\varepsilon(1)$, as given by

$$
\varepsilon(1)=\varepsilon(\infty) \prod_{i=3}^{N} \frac{\omega_{\mathrm{LO} i}^{2}}{\omega_{\mathrm{TO} i}^{2}} .
$$

In BIT, $\varepsilon(\omega)$ is anisotropic and depends on the polarization direction. For light polarization parallel to the $a$ axis $(E \| a)$ and the $b$ axis $(E \| b)$, we studied $\varepsilon_{a}(\omega)$ and $\varepsilon_{b}(\omega)$, respectively. For the two directions, two different low-frequency polariton branches were separately observed down to 3 $\mathrm{cm}^{-1}$. The solid curves in Figs. 5(a) and 5(b) denote the curves calculated by Eq. (2) using mode frequencies of $A^{\prime}(x, z)$ and $A^{\prime \prime}(y)$ symmetries, respectively. There was good agreement between the observed and calculated polariton-dispersions. Therefore, it is concluded that this nonlinear relation reflects the dispersion relation of bulk phonon-polariton. Table I, shows the values of fitting parameters for observed polariton-dispersions.

\section{DISCUSSION}

Up to now, phonon-polariton has been studied mainly by forward Raman scattering experiments. ${ }^{14,15}$ The problem was the observation of low-frequency polariton below $100 \mathrm{~cm}^{-1}$ because the intense elastic scattering causes the strong Rayleigh wings which screen the polariton peak from Raman spectra. Although the lower limit had been markedly reduced upon the use of a Raman spectrometer with the highest stray light rejection of $10^{-14}$ at $20 \mathrm{~cm}^{-1}$, the observation below 20 $\mathrm{cm}^{-1}$ was still difficult. ${ }^{16}$ Since 1992 , several different techniques for the observation of phonon-polariton were introduced such as impulsive stimulated Raman scattering (ISRS) with femtosecond laser pulses. ${ }^{17-20}$ However, ISRS measurements on $\mathrm{LiTaO}_{3}$ and $\mathrm{LiNbO}_{3}$ resulted in conflicting results concerning splitting in the low-frequency polariton branches, ${ }^{20-22}$ whereas no such splitting is observed here.
Finally we discuss the advantages of THz-TDS for investing low-frequency polariton. THz-TDS has four important advantages compared with previous methods. First, since the incident beam is not intense visible light but rather weak $\mathrm{THz}$ radiation, no optical damage occurs in colored or opaque samples. Second, concerning extraordinary polaritons of which Raman tensor is off-diagonal, other scattering methods have serious problems. Since the polarization planes of incident and scattered beams are orthogonal for extraordinary polaritons, these methods cannot cover very low frequencies due to birefringence which causes the unavoidable finite difference of wave vectors between the incident and scattering beams even if the scattering angle goes to zero. Therefore, in the low-frequency region, only ordinary polariton was studied by other methods, i.e., Raman scattering, ISRS and CARS, whereas, by THz-TDS, we can easily measure polariton frequency down to a few wave numbers, even for extraordinary polaritons. Third, we can measure the $k$ dependence minutely without changing the optical geometry, and the uncertainty of $k$ is very small especially for small $k$. In contrast, with Raman scattering, $k$ is determined by a scattering angle, and the uncertainty increases as $k$ approaches zero. Fourth, THz-TDS can be applied to Raman inactive but IR-active modes, especially to ferroelectric soft modes in paraelectric phases.

\section{CONCLUSIONS}

We studied phonon-polariton by using THz-TDS. The frequency dependence of the transmission phase delay was accurately measured for a high-quality ferroelectric bismuth titanate crystal. The obtained phonon-polariton dispersion relation was well reproduced using the calculation based on Kurosawa's formula. The lowest branch of phonon-polariton dispersion was determined down to the very low frequency of $3 \mathrm{~cm}^{-1}$, which cannot be attained by other optical experimental methods, especially for extraordinary polaritons. Therefore, we believe that this is the first observation of the dispersion relation of phonon-polariton in the far-infrared region by THz-TDS. The anisotropy of polariton dispersion was also clearly observed simply by rotating the polarization plane of the incident beam. The present measurement also indicates the possibility of accurate determination of the damping factor of the phonon-polariton in the far-infrared region by using the $k$ dependence of transmission and reflection amplitude.

\section{ACKNOWLEDGMENTS}

We thank Professor K. Sakata of Tokyo Science University for providing the bismuth titanate single crystal. This work was supported in part by the Japan Science and Technology Corporation.
*Email address: kojima@bk.tsukuba.ac.jp

†Present address: Tochigi Nikon Corp., Ohtawara, Tochigi 3248625, Japan.

${ }^{1}$ M. van Exter and D. Grischkowsky, Appl. Phys. Lett. 56, 1694

(1990).
${ }^{2}$ L. Thrane, R. H. Jacobsen, P. U. Jepsen, and S. R. Keiding, Chem. Phys. Lett. 240, 330 (1995).

${ }^{3}$ J. T. Kindt and C. A. Schmuttenmaer, J. Phys. Chem. 100, 10373 (1996).

${ }^{4}$ R. Harel, I. Brener, L. N. Pfeiffer, K. W. West, J. M. Vandenberg, 
S. G. Chu, and J. D. Wynn, Phys. Status Solidi A 178, 365 (2000)

${ }^{5}$ M. Wada, Y. Doi, K. Inoue, J. W. Haus, and Z. Yuan, Appl. Phys. Lett. 70, 2966 (1997).

${ }^{6}$ T. Aoki, M. Wada, J. W. Haus, Z. Yuan, M. Tani, K. Sakai, N. Kawai, and K. Inoue, Phys. Rev. B 64, 045106 (2001).

${ }^{7}$ C. A. Arajo, J. D. Cuchiaro, M. C. Scott, and L. D. MacMillan, Nature (London) 374, 627 (1995).

${ }^{8}$ J. F. Scott, Phys. Today 51 (7), 22 (1998).

${ }^{9}$ E. C. Subarao, J. Phys. Chem. Solids 23, 665 (1962).

${ }^{10}$ S. Kojima and S. Shimada, Physica B 219\&220, 617 (1996).

${ }^{11}$ K. Huang, Proc. R. Soc. London, Ser. A 208, 352 (1951).

${ }^{12}$ Max Born and Kun Huang, Dynamical Theory of Crystal Lattice (Oxford University Press, Oxford, 1954), Chap. II.8.

${ }^{13}$ T. Kurosawa, J. Phys. Soc. Jpn. 16, 1298 (1961).

${ }^{14}$ C. H. Henry and J. J. Henry, Phys. Rev. Lett. 15, 964 (1965).
${ }^{15}$ R. Claus, L. Merten, and J. Brandmuller, Light Scattering by Phonon-Polaritons, Springer Tracts in Modern Phys. Vol. 75, (Springer-Verlag, Berlin, 1975).

${ }^{16}$ S. Kojima and T. Nakamura, Jpn. J. Appl. Phys. 19, L609 (1980).

${ }^{17}$ P. C. M. Planken, L. D. Noordam, J. T. M. Kennis, and A. Lagendijk, Phys. Rev. B 45, 7106 (1992).

${ }^{18}$ H. J. Bakker, S. Hunsche, and H. Kurtz, Phys. Rev. B 48, 13524 (1993).

${ }^{19}$ H. J. Bakker, S. Hunsche, and H. Kurtz, Phys. Rev. B 50, 914 (1994).

${ }^{20}$ G. P. Wiederrecht, T. P. Dougherty, L. Dhar, and K. A. Nelson, Phys. Rev. B 51, 916 (1995).

${ }^{21}$ T. Watanabe, S. Yoshioka, M. Kasahara, K. A. Nelson, T. F. Grimmins, and T. Yagi, J. Korean Phys. Soc. 35, S1400 (1999).

${ }^{22}$ S. Kojima, Ferroelectrics 223, 63 (1999). 